\title{
Perfluorooctane sulfonate induces neuronal and oligodendrocytic differentiation in neural stem cells and alters the expression of PPAR $\gamma$ in vitro and in vivo
}

\begin{abstract}
Perfluorinated compounds are ubiquitous chemicals of major concern for their potential adverse effects on the human population. We have used primary rat embryonic neural stem cells (NSCs) to study the effects of perfluorooctane sulfonate (PFOS) on the process of NSC spontaneous differentiation. Upon removal of basic fibroblast growth factor, NSCs were exposed to nanomolar concentrations of PFOS for $48 \mathrm{~h}$, and then allowed to differentiate for additional 5 days. Exposure to 25 or $50 \mathrm{nM}$ concentration resulted in a lower number of proliferating cells and a higher number of neurite-bearing TuJ1-positive cells, indicating an increase in neuronal differentiation. Exposure to $50 \mathrm{nM}$ also significantly increased the number of CNPase-positive cells, pointing to facilitation of oligodendrocytic differentiation. PPAR genes have been shown to be involved in PFOS toxicity. By q-PCR we detected an upregulation of PPAR $\gamma$ with no changes in PPAR $\alpha$ or PPAR $\delta$ genes. One of the downstream targets of PPARs, the mitochondrial uncoupling protein 2 (UCP2) was also upregulated. The number of TuJ1- and CNPase-positive cells increased after exposure to PPAR $\gamma$ agonist rosiglitazone (RGZ, $3 \mu \mathrm{M})$ and decreased after pre-incubation with the PPAR $\gamma$ antagonist GW9662 $(5 \mu \mathrm{M})$. RGZ also upregulated the expression of PPAR $\gamma$ and UCP2 genes. Meanwhile GW9662 abolished the UCP2 upregulation and decreased Ca2 + activity induced by PFOS. Interestingly, a significantly higher expression of PPAR $\gamma$ and UCP3 genes was also detected in mouse neonatal brain after prenatal exposure to PFOS. These data suggest that PPAR $\gamma$ plays a role in the alteration of spontaneous differentiation of NSCs induced by nanomolar concentrations of PFOS.
\end{abstract}

Keyword: Calcium; Differentiation; Neural stem cells; PFOS; PPAR; Proliferation. 